\title{
Investigating Topological Insulators with High Harmonics
}

\author{
Unlike most conventional materials, topological insulators generate \\ harmonics when driven with circularly polarized lasers, which opens a \\ window on their surface electronic properties.
}

By Erika K. Carlson

$$
\text { a }
$$
$\mathrm{n}$ the past decade, researchers have tried a variety of techniques to understand the peculiar properties of topological insulators-exotic materials with conductive surfaces but insulating interiors. A recent approach involves an optical technique called high harmonics generation (HHG). Now, researchers at SLAC National Accelerator Laboratory in California have modeled the process of HHG in the topological insulator bismuth selenide, predicting that the material's surface and bulk should react differently to linearly and circularly polarized light [1]. The work suggests a new way to probe topological insulators and their exotic behaviors.

In $\mathrm{HHG}$, an intense laser pulse interacts with a medium to produce high harmonics-light at multiples of the input frequency. In bismuth selenide, the surface and bulk each generate harmonics with distinct characteristics. The researchers used this discrepancy to identify the factors that determine the harmonics generated by topological insulators in

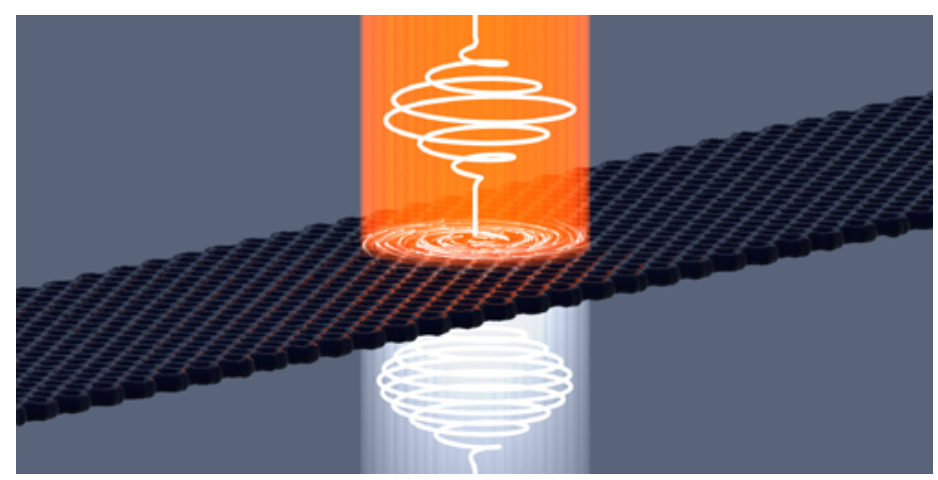

Credit: G. Stewart/SLAC National Accelerator Laboratory general.

High harmonics are generated in isotropic media and most conventional materials by linearly polarized light but not by circularly polarized light. The researchers found that the bulk of bismuth selenide follows this rule. But they also found that the material's surface produces harmonics effectively when the input laser beam is circularly polarized. The researchers say that this surprising behavior likely results from light-induced circular motion of electrons on the topological insulator's surface. Further studies of such interactions may lead to improved understanding of these materials' exotic surface electronic properties. These studies may even allow researchers to induce topological properties in "normal" materials by applying an appropriate light field.

Erika K. Carlson is a Corresponding Editor for Physics based in New York City.

\section{REFERENCES}

1. D. Baykusheva et al., "Strong-field physics in three-dimensional topological insulators," Phys. Rev. A 103, 023101 (2021). 